\title{
Corrigendum
}

Sandra Carillo

To cite this article: Sandra Carillo (2015) Corrigendum, Journal of Nonlinear Mathematical Physics 12:Supplement 1, i-iii, DOI:

https://doi.org/10.1080/14029251.2014.971573

To link to this article: https://doi.org/10.1080/14029251.2014.971573

Published online: 04 January 2021 


\title{
CORRIGENDUM
}

\author{
of
}

\section{Some remarks on materials with memory: heat conduction and viscoelasticity}

Sandra CARILLO

Throughout there are 16 missing "=" signs. Here follows a list of corrected formulas:

1. formula (2.2) page $165(1$ "=" sign missing)

- ORIGINAL

- CORRECTED

$$
\begin{gathered}
\mathbf{q}(\mathbf{x}, t)-\int_{0}^{\infty} k(\mathbf{x}, \tau) \nabla \theta(\mathbf{x}, t-\tau) d \tau \\
\mathbf{q}(\mathbf{x}, t)=-\int_{0}^{\infty} k(\mathbf{x}, \tau) \nabla \theta(\mathbf{x}, t-\tau) d \tau,
\end{gathered}
$$

2. formula (2.6) page 165 (2 "=" signs missing)

- ORIGINAL

$$
\mathbf{q}(t)-\int_{0}^{\infty} k(\tau) \mathbf{g}(t-\tau) d \tau \quad \text { or } \quad \mathbf{q}(t) \int_{0}^{\infty} \dot{k}(\tau) \overline{\mathbf{g}}^{t}(\tau) d \tau
$$

- CORRECTED $\mathbf{q}(t)=-\int_{0}^{\infty} k(\tau) \mathbf{g}(t-\tau) d \tau \quad$ or $\quad \mathbf{q}(t)=\int_{0}^{\infty} \dot{k}(\tau) \overline{\mathbf{g}}^{t}(\tau) d \tau$

3. formula (2.7) page $166(1$ "=" sign missing)

- ORIGINAL

- CORRECTED

$$
\begin{array}{r}
\overline{\mathbf{g}}^{t}(\tau) \int_{t-\tau}^{t} \mathbf{g}(s) d s \\
\overline{\mathbf{g}}^{t}(\tau)=\int_{t-\tau}^{t} \mathbf{g}(s) d s
\end{array}
$$

4. formula (2.10) page 166 , on the r.h.s. (1 "=" sign missing)

- ORIGINAL

$$
\widetilde{Q}\left\{\overline{\mathbf{g}}^{t}\right\}:=\int_{0}^{\infty} \dot{k}(s) \overline{\mathbf{g}}^{t}(s) d s \Longrightarrow \forall T>0, \quad \widetilde{Q}\left\{\overline{\mathbf{g}}^{t+T}\right\} \int_{0}^{\infty} \dot{k}(s) \overline{\mathbf{g}}^{t+T}(s) d s,
$$

- CORRECTED

$$
\widetilde{Q}\left\{\overline{\mathbf{g}}^{t}\right\}:=\int_{0}^{\infty} \dot{k}(s) \overline{\mathbf{g}}^{t}(s) d s \Longrightarrow \forall T>0, \quad \widetilde{Q}\left\{\overline{\mathbf{g}}^{t+T}\right\}=\int_{0}^{\infty} \dot{k}(s) \overline{\mathbf{g}}^{t+T}(s) d s,
$$


5. formula (2.11) page 166 ( 1 “=” sign missing)

- ORIGINAL

CORRECTED

$$
\begin{aligned}
\mathbf{q}(t+T) & : \int_{0}^{\infty} \dot{k}(s) \overline{\mathbf{g}}^{t+T}(s) d s . \\
\mathbf{q}(t+T) & :=\int_{0}^{\infty} \dot{k}(s) \overline{\mathbf{g}}^{t+T}(s) d s .
\end{aligned}
$$

6. formula (2.12) page 166 ( 1 "=" sign missing)

- ORIGINAL

$$
\theta(t) \theta_{\star}(0)+\int_{0}^{t} \dot{\theta}_{P}(\xi) d \xi \quad, \quad \overline{\mathbf{g}}^{t}(s)= \begin{cases}\int_{t-s}^{t} \mathbf{g}_{P}(\xi) d \xi & 0 \leq s<t \\ \int_{0}^{t} \mathbf{g}_{P}(\xi) d \xi+\overline{\mathbf{g}}_{\star}^{0}(s-t) & s \geq t\end{cases}
$$

\section{- CORRECTED}

$$
\theta(t)=\theta_{\star}(0)+\int_{0}^{t} \dot{\theta}_{P}(\xi) d \xi \quad, \quad \overline{\mathbf{g}}^{t}(s)= \begin{cases}\int_{t-s}^{t} \mathbf{g}_{P}(\xi) d \xi & 0 \leq s<t \\ \int_{0}^{t} \mathbf{g}_{P}(\xi) d \xi+\overline{\mathbf{g}}_{\star}^{0}(s-t) & s \geq t,\end{cases}
$$

7. formula (3.9) page 169 (1 "=" sign missing)

$$
\begin{array}{ll}
\text { - ORIGINAL } & \langle f, \phi\rangle: \int_{0}^{+\infty} f(t) \cdot \phi(t) d t=\frac{1}{2 \pi} \int_{-\infty}^{+\infty} f_{+}(\omega) \cdot \overline{\phi_{+}(\omega)} d \omega, \\
\text { - CORRECTED } & \langle f, \phi\rangle:=\int_{0}^{+\infty} f(t) \cdot \phi(t) d t=\frac{1}{2 \pi} \int_{-\infty}^{+\infty} f_{+}(\omega) \cdot \overline{\phi_{+}(\omega)} d \omega,
\end{array}
$$

8. formula (3.12) page $169(1$ "=" sign missing)

$$
\begin{aligned}
& \text { - ORIGINAL } \mathcal{H}\left(\mathbb{R}^{+}, \mathbb{R}^{3}\right):\left\{\phi: \mathbb{R}^{+} \rightarrow \mathbb{R}^{3}:\left|\int_{-\infty}^{+\infty} k_{c}(\omega) \phi_{+}(\omega) \cdot \overline{\phi_{+}(\omega)} d \omega\right|<\infty\right\} . \\
& \text { - CORRECTED } \mathcal{H}\left(\mathbb{R}^{+}, \mathbb{R}^{3}\right):=\left\{\phi: \mathbb{R}^{+} \rightarrow \mathbb{R}^{3}:\left|\int_{-\infty}^{+\infty} k_{c}(\omega) \phi_{+}(\omega) \cdot \overline{\phi_{+}(\omega)} d \omega\right|<\infty\right\} .
\end{aligned}
$$

9. formula (3.13) page $170(1$ "=" sign missing)

$$
\begin{array}{lr}
\text { - ORIGINAL } & \langle f, \phi\rangle_{k}: \int_{-\infty}^{+\infty} k_{c}(\omega) f_{+}(\omega) \cdot \overline{\phi_{+}(\omega)} d \omega \\
\text { - CORRECTED } & \langle f, \phi\rangle_{k}:=\int_{-\infty}^{+\infty} k_{c}(\omega) f_{+}(\omega) \cdot \overline{\phi_{+}(\omega)} d \omega
\end{array}
$$

10. formula (3.15) page $170(1 “="$ sign missing $)$
- ORIGINAL
$\langle\mathbf{I}, \mathbf{g}\rangle: \frac{1}{2 \pi} \int_{-\infty}^{+\infty} \mathbf{I}_{+}(\omega) \cdot \overline{\mathbf{g}_{+}(\omega)} d \omega$
- CORRECTED
$\langle\mathbf{I}, \mathbf{g}\rangle:=\frac{1}{2 \pi} \int_{-\infty}^{+\infty} \mathbf{I}_{+}(\omega) \cdot \overline{\mathbf{g}_{+}(\omega)} d \omega$ 
11. formula (3.16) page $170(1$ "=" sign missing)

- ORIGINAL $\mathcal{H}_{k}^{\prime}\left(\mathbb{R}^{+}, \mathbb{R}^{3}\right):\left\{f: \mathbb{R}^{+} \rightarrow \mathbb{R}^{3}\right.$ s.t. $\left.\left|\langle f, \phi\rangle_{k}\right|<\infty, \forall \phi \in \mathcal{H}_{k}\left(\mathbb{R}^{+}, \mathbb{R}^{3}\right)\right\}$, - CORRECTED

$$
\mathcal{H}_{k}^{\prime}\left(\mathbb{R}^{+}, \mathbb{R}^{3}\right):=\left\{f: \mathbb{R}^{+} \rightarrow \mathbb{R}^{3} \text { s.t. }\left|\langle f, \phi\rangle_{k}\right|<\infty, \forall \phi \in \mathcal{H}_{k}\left(\mathbb{R}^{+}, \mathbb{R}^{3}\right)\right\},
$$

12. formula (3.20) page 171 , on the r.h.s. (1 "=" sign missing)

$\begin{array}{lcl}\text { - ORIGINAL } & \mathcal{W}\left(\sigma(t), \mathbf{g}_{P}\right):=\widetilde{W}\left\{\overline{\mathbf{g}}^{t} ; \mathbf{g}_{P}\right\} & \Longleftrightarrow \mathcal{W}\left(\sigma(t), \mathbf{g}_{P}\right) \widehat{W}\left\{\overline{\mathbf{g}}^{t} ; \mathbf{g}_{P}\right\} . \\ \text { - CORRECTED } & \mathcal{W}\left(\sigma(t), \mathbf{g}_{P}\right):=\widetilde{W}\left\{\overline{\mathbf{g}}^{t} ; \mathbf{g}_{P}\right\} \Longleftrightarrow \mathcal{W}\left(\sigma(t), \mathbf{g}_{P}\right)=\widehat{W}\left\{\overline{\mathbf{g}}^{t} ; \mathbf{g}_{P}\right\} .\end{array}$

13. formula (6.1) page 176 ( $2 "="$ signs missing)

- ORIGINAL $f_{s}(\omega) \int_{0}^{\infty} f(\tau) \sin \omega \tau d \tau, f_{c}(\omega) \int_{0}^{\infty} f(\tau) \cos \omega \tau d \tau$,

- CORRECTED $f_{s}(\omega)=\int_{0}^{\infty} f(\tau) \sin (\omega \tau) d \tau, f_{c}(\omega)=\int_{0}^{\infty} f(\tau) \cos (\omega \tau) d \tau$,

14. formula (6.3) page 176 , on the 1.h.s. (1 "=" sign missing)

- ORIGINAL $\tilde{f}(\omega) \int_{-\infty}^{\infty} f(\tau) e^{-i \omega \tau} d \tau$ hence $\tilde{f}(\omega)=f_{c}(\omega)-i f_{s}(\omega)$,

- CORRECTED $\tilde{f}(\omega)=\int_{-\infty}^{\infty} f(\tau) e^{-i \omega \tau} d \tau$ hence $\tilde{f}(\omega)=f_{c}(\omega)-i f_{s}(\omega)$ 